Jpn. J. Med. Sci. Biol., 1992

\title{
3. Summary of Reported Isolations of Viruses (including Rickettsiae, Chlamydiae and Mycoplasmas), 1991
}

3-1. Isolation of viruses, rickettsiae, chlamydiae and mycoplasmas from humans

\section{Overview}

In 1991, echovirus 30 (E30) caused an extra large nationwide epidemic of aseptic meningitis in Japan. The virus prevailed for three successive years since 1989, and in the third year, 1991, the reported isolations of E30 recorded the largest number of the single virus type on yearly basis ever reported.

A total of 13,452 virus isolates from humans was reported for the year 1991 (reports updated on November 30, 1992) by 62 institutions (Fig. 20). This was $126.7 \%$ of the previous year's total (reports for the year 1990 updated on September $30,1991)$.

The virus reporting system included three types of collaborating institutions: prefectural/municipal public health institutes (PHI), university/national hospitals and private clinical laboratories. $\mathrm{PHI}$ reported 10,846 isolations, accounting for $80.6 \%$ of all virus reports received for the year 1991, while university/national hospitals reported 796 isolations, or 5.9\%; private clinical laboratories reported 1,810 isolations, or $13.5 \%$. Among the isolations in $1991,7,555$ were from specimens collected by sentinel hospitals and clinics under the program of the National Epidemiological Surveillance of Infectious Diseases; this represents $56.2 \%$ of the year's reports, compared with $57.4 \%$ in the previous year.

The increase in the total number of isolations in 1991 from the previous year was mainly due to the increased number of E30. In 1991, the total number of enterovirus isolations was 6,097 representing $165 \%$ of the previous year's total. E30 reports numbered 4,059 accounting for $66.6 \%$ of the total enterovirus, and $30.2 \%$ of total virus isolation reports in 1991. Reports of coxsackievirus $A$ and coxsackievirus $B$ were $87.7 \%$ and $46.1 \%$ of the respective numbers of the previous year's reports. Coxsackievirus A16 and enterovirus 71, both increased in the previous year, decreased markedly in 1991. For influenza viruses, A (H3N2) (2,051 isolations) increased but type $B$ (579 isolations) decreased from the previous year. In addition, $A(H 1 N 1)$ which was not isolated in the previous year increased to 308 reports. Rotavirus reports were almost the same level (574 isolations) as the 1990 's figure and less than half of the 1989's reports. Adenovirus reports were 1,864 , representing $116.4 \%$ of the previous year's figure. 
Vol. 45, Suppl.

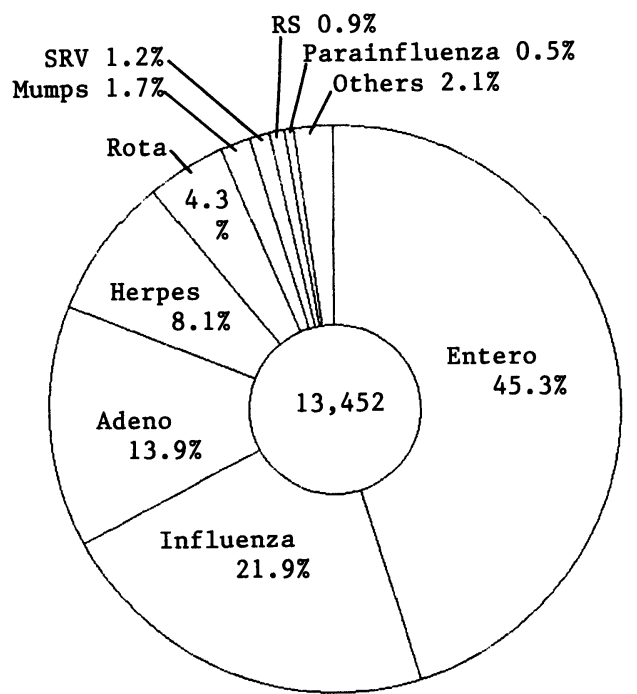

Fig. 20. Reported isolations of viruses from humans, 1991

In 1991, two Rickettsia tsutsugamushi (R. tsutsugamushi) isolations from human sources were reported. Mycoplasma pneumoniae (M. pneumoniae) reports were only two, reflecting periodical decrease after the 1988-epidemic of atypical pneumonia due to this organism. Reports of Chlamydia were 376 cases, nearly the same level as those in previous three years.

\section{Enteroviruses}

The total number of isolations of enteroviruses in 1991 was 6,097. Among them, 4,128 (67.7\%) were isolations from specimens obtained through the sentinel hospitals and clinics collaborating under the National Epidemiological Surveillance of Infectious Diseases.

Among enterovirus groups, echovirus (E) group was overwhelming, accounting for $76.0 \%$ of enterovirus isolations in 1991, followed by coxsackie $A$ (CA) group accounting for $15.4 \%$, and coxsackie B (CB) group accounting for $6.5 \%$ (Fig. 21). Most frequently reported enterovirus serotypes were E30 (4,059 isolations), followed by, in much lower number, E9 (350), CA2 and CA6, which accounted for $66.6 \%, 5.7 \%, 4.4 \%$ and $4.2 \%$, respectively, of the total number of enteroviruses (Fig. 22). 
Jpn. J. Med. Sci. Biol., 1992

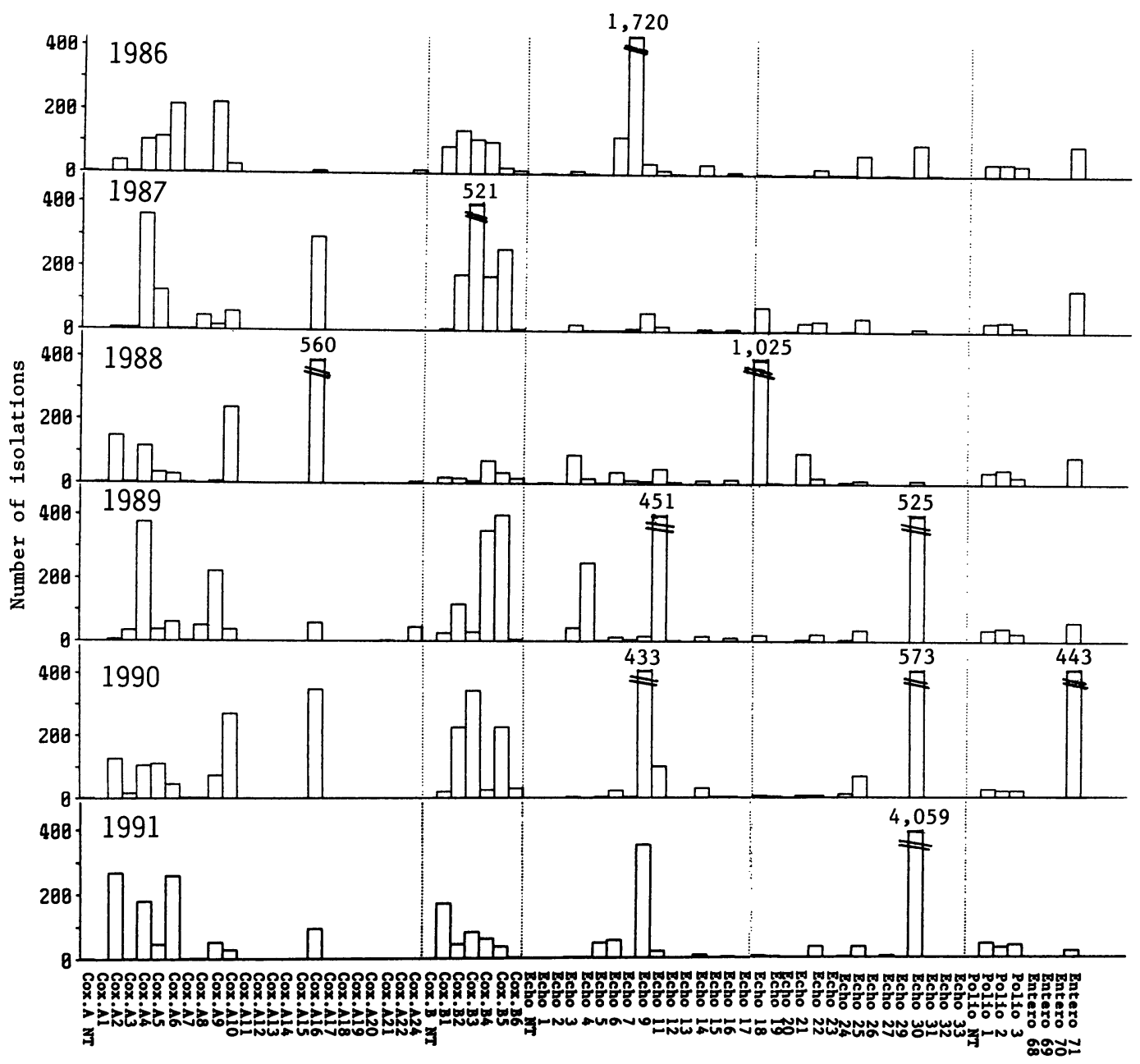

Fig. 21. Reported isolations of enteroviruses from humans, 1986-1991

In 1991, the total number of reports of coxsackie $A$ viruses was 938 , which was $87.7 \%$ of the previous year's figure, 1,070. Reports of CA16, the major causative enterovirus of hand-foot-and-mouth disease, decreased to 94 from the previous year's 347 . Of these, $78(83.0 \%)$ implicated hand-foot-and-mouth disease and one meningitis. 
Vol. 45, Suppl.

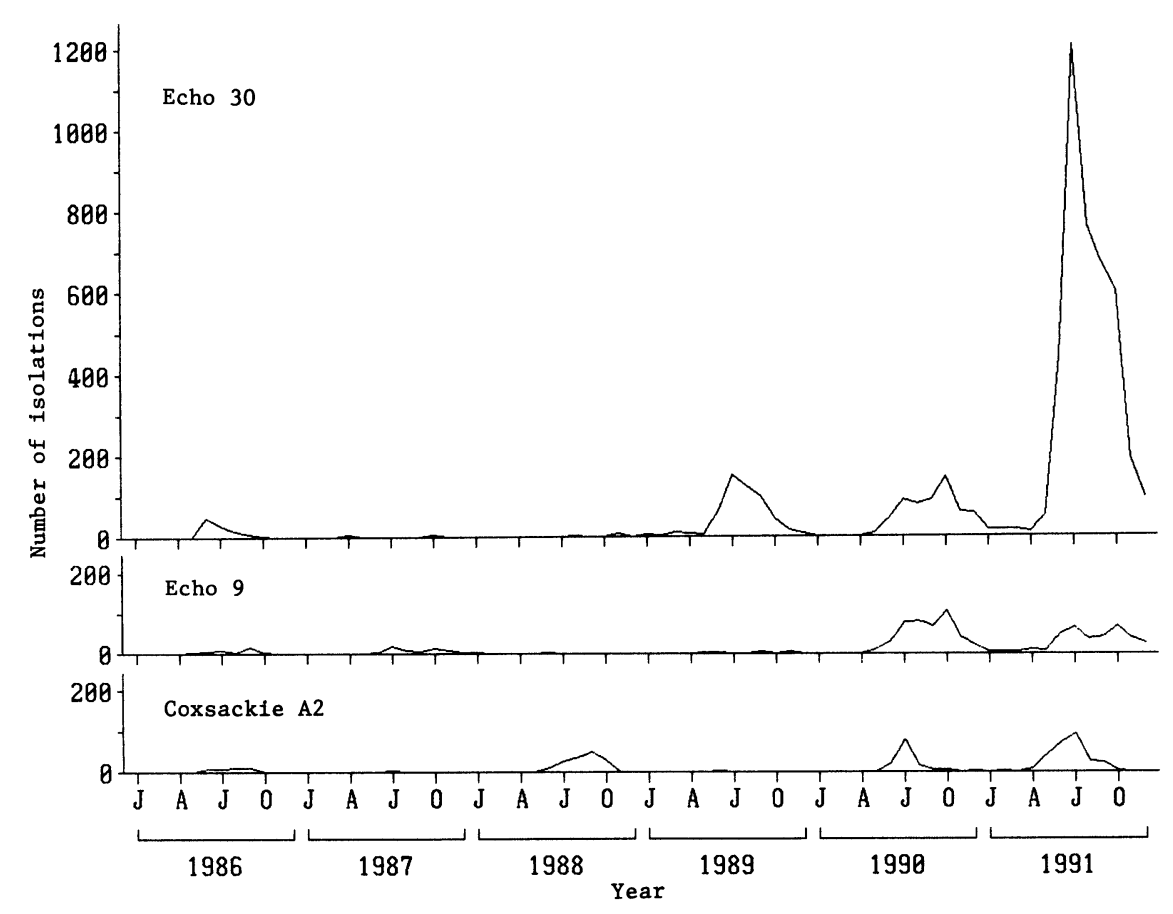

Fig. 22. Reported isolations of 1991's top three enteroviruses from humans, by month, 1986-1991

Isolation of enterovirus (EV) 71, which is another causative virus of handfoot-and-mouth disease, also markedly decreased to 21 . Of these, hand-foot-andmouth disease was reported in $19(90.5 \%)$ and meningitis in one.

CA virus types 1-10 (except type 9) are the main cause of herpangina, and a few types of them increase every year in various combinations. In 1991, reports of CA2, CA4 and CA6 increased to 269, 181 and 258, respectively. The herpanginaassociation frequency of these CA viruses ranged from 50.8 to $63.3 \%$. Central nervous system complications other than meningitis were reported in two CA6 and one CA9 cases (Table).

CB viruses have been prevailing every year, though the prevalent types change from year to year. Total isolations, involving six CB types, in 1991 numbered 394 , accounting for $46.1 \%$ of the previous year's figure; this represented $6.5 \%$ of the total enterovirus isolations in 1991 . The frequently isolated types were CB1 (170 isolations); reports of the other five types ranged from 80 to four. Among the total CB virus isolations with clinical records, meningitis was reported in 23.2\% (84/362) and encephalitis in two (Table). CB virus isolations from CSF were reported in $64(16.2 \%)$. 
Jpn. J. Med. Sci. Biol., 1992

\section{Table. Viruses isolated from cases with clinical syndrome of central nervous system other than aseptic meningitis, Japan, 1991}

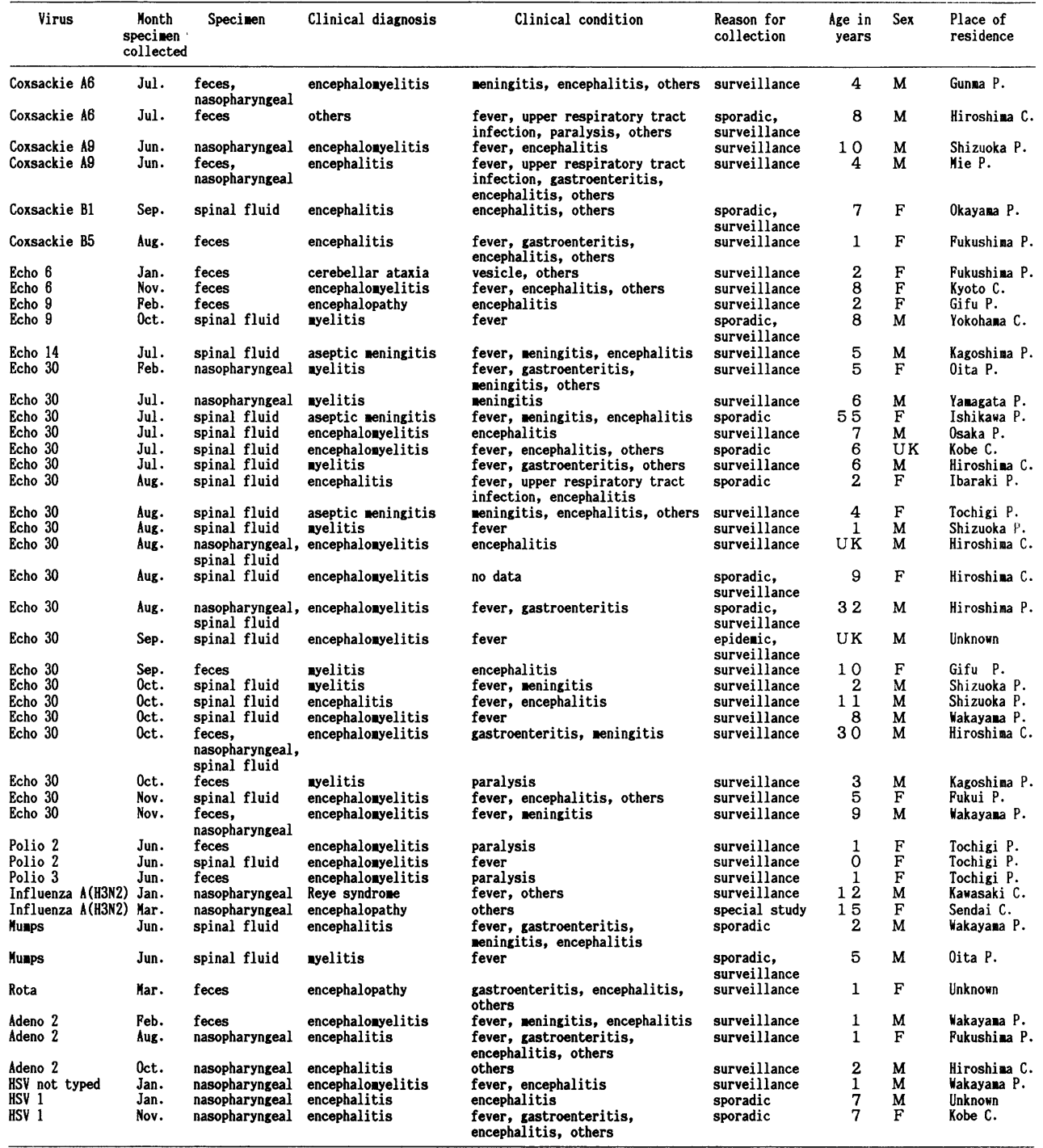

* Includes the cases associated with encephalonyelitis, encephalitis, melitis, paralysis, encephalopathy, Reye syndrone, cerebellar ataxia UK : Unknown, P: Prefecture, C: City 


\section{Vol. 45, Suppl.}

The total number of echovirus isolations was 4,631, representing 3.7 times of the previous year's figure. Of these, $4,059(87.6 \%)$ were E30 and 350 were E9 which was the second most frequently reported echovirus.

E9 isolations increased for successive two years (Fig. 22); they were 431 reports in 1990. Among $263 \mathrm{E} 9$ reports in 1991 with clinical information, 158 (60.1\%) were associated with meningitis, 61 (23.2\%) with exanthem, one each with encephalopathy and myelitis (Table). E9 isolations from CSF were reported in 139 cases $(39.7 \%)$.

E30, which was prevalent in the previous two years, again increased in the summer of 1991 (Fig 22), with the number not only much larger than the previous two years but also the largest on yearly basis for the single virus type in the past. E30 was the virus type most frequently associated with meningitis, which represent 2,637 (75.7\%) of 3,484 E30 reports with clinical information. On the other hand, only $57(1.6 \%)$ were associated with exanthem. Central nervous system involvement other than meningitis were reported in 21 cases (Table). $\quad$ E30 isolations from CSF were reported in 2,457 cases $(60.5 \%)$.

The majority of enterovirus isolations were from young children. Those at 0 4 years of age were in much higher proportion than those at 5-9 years of age; they were $74.9 \%$ vs. $22.2 \%$ of total CA isolations, $61.7 \%$ vs. $31.8 \%$ of total CB isolations and also $52.3 \%$ vs. $42.5 \%$ of E9 isolations, respectively, in 1991 . On the contrary, E30 was more frequently isolated from older children: isolations from 0-4 years of age were $36.4 \%$, while from 5 to 9 -year-olds were $52.4 \%, 10$ to 14 -year-olds were $8.8 \%$ and those aged from 15 years to 60 s years were $2.4 \%$ (96 cases). The age distributions of these viruses were nearly the same as in the previous year.

The most common source of enteroviruses was the nasopharyngeal specimen. Namely, the rate of isolation from nasopharyngeal materials was $48.5 \%$ of viruspositive cases, while that from feces was $30.9 \%$ and that from CSF was $44.7 \%$ (sometimes virus was recovered from two or more specimens of the same person). The proportion from CSF was much higher in 1991 than previous years due to the extremely increased number of E30 reports with aseptic meningitis.

Enteroviruses were isolated in cell culture or mice. The frequency of isolation of most CA viruses in mice ( $>83 \%$ ) was much higher than that in cell culture (<23\%) for CA types 2, 4, 5, and 6 in 1991 (including specimens that were virus-positive both in mice and in cell culture). However, CA9 and CA16 were preferentially recovered in cell culture (100\% and $91.5 \%$, respectively). All CB isolates (of these, one was isolated in duplicate in mice) and all strains of 


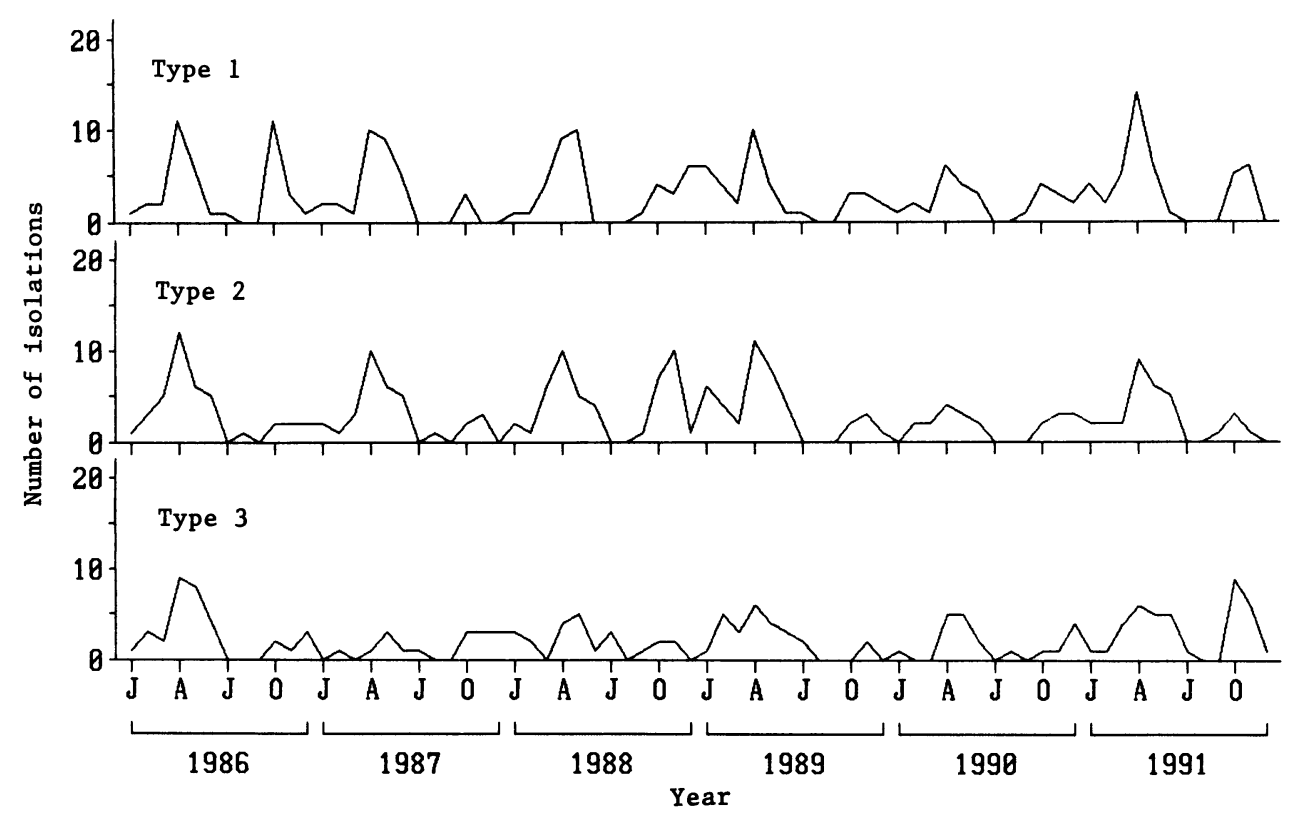

Fig. 23. Reported isolations of polioviruses from humans, by month, 1986-1991

echovirus, EV71 and poliovirus were isolated in cell culture.

Enterovirus isolation usually peaks in the summer, but it often continued even during the winter on a low level. E30 isolation peaked in June in 1991.

For polioviruses, seasonal distribution showed two peaks, one in spring and the other in autumn, as previously, coinciding with the time of administration of the live oral polio vaccine in Japan (Fig. 23). In 1991, two cases with encephalomyelitis were reported (Table); one with isolation from feces of poliovirus types 2 and 3 simultaneously, and the other type 2 from CSF. In addition, from another one, isolation of type 1 from CSF was reported. All poliovirus isolates from the cases with central nervous system involvement were identified as the vaccine strains.

\section{Rhinovirus}

In 1991, 30 isolations of rhinovirus were reported from Sendai National Hospital throughout the year except three months, August, November and December. 
Vol. 45, Suppl.

\section{Influenza virus}

The number of influenza virus isolations reported in 1991 was 2,943 in total, including 308 isolates of type A (H1N1), 2,051 of A (H3N2), 579 of type B and four of type C. This annual report includes the isolation from two independent influenza seasons, January-June, 1991 (the main part of the 1990/91 epidemic) and November-December, 1991 (the early part of the 1991/92 epidemic).

In the 1990/91 influenza season in Japan, influenza A(H3N2) predominantly prevailed, together with types B and A (H1N1) in lesser extent. A(H3N2) epidemic started in December, 1990, peaking in February, 1991, and continued until May with 2,044 isolations in total; whereas, type $B$ isolations were reported from January through June, 1991, peaking in March with 579 isolations. The number of A(H1N1) isolations was 248 in January-June with a peak in March.

The influenza virus prevalence in the 1991/92-influenza season started in November, 1991; 59 A(H1N1) strains were isolated in November-December, while six A(H3N2) were in December. These isolations followed by the influenza epidemic in early 1992 , in which $\mathrm{A}(\mathrm{H} 1 \mathrm{~N} 1)$ was the most frequently reported type and $A(H 3 N 2)$ was the next (Fig. 24).

Isolations of influenza viruses were reported from patients of all ages. Of the reports including age information in 1991, isolation from children 10-14 years old was most frequent for $\mathrm{A}(\mathrm{H} 3 \mathrm{~N} 2)$ accounting for $51.9 \%$ of cases and 5-9 years old was $24.3 \%$, while those from $5-9$ years old were most frequent for $\mathrm{A}(\mathrm{H} 1 \mathrm{~N} 1)$ and type $\mathrm{B}$, accounting for $43.2 \%$ and $48.0 \%$, respectively.

All influenza viruses were isolated from nasopharyngeal specimens except for the two which were from eye specimens. Isolation was done in cell culture or chicken embryonated eggs. Reports of isolations in cell culture were more frequent than in embryonated eggs: 306 isolations (99.4\% of virus-positive cases) were in cell culture and $12(3.9 \%)$ in embryonated eggs for A (H1N1), 1,811 (88.3\%) and $336(16.4 \%)$ for $\mathrm{A}$ (H3N2), and $560(96.7 \%)$ and $23(4.0 \%)$ for type B, respectively, in 1991.

Of patients infected with influenza viruses, those with lower respiratory tract illness/pneumonia were $7.2 \%$ for $A$ (H1N1), $5.1 \%$ for $A(H 3 N 2)$ and $5.1 \%$ for type B.

\section{Parainfluenza virus}

A total of 73 reports of parainfluenza viruses were received from nine institutions, representing $96.1 \%$ of the previous year's figure: Of these, 48 were 
Jpn. J. Med. Sci. Biol., 1992

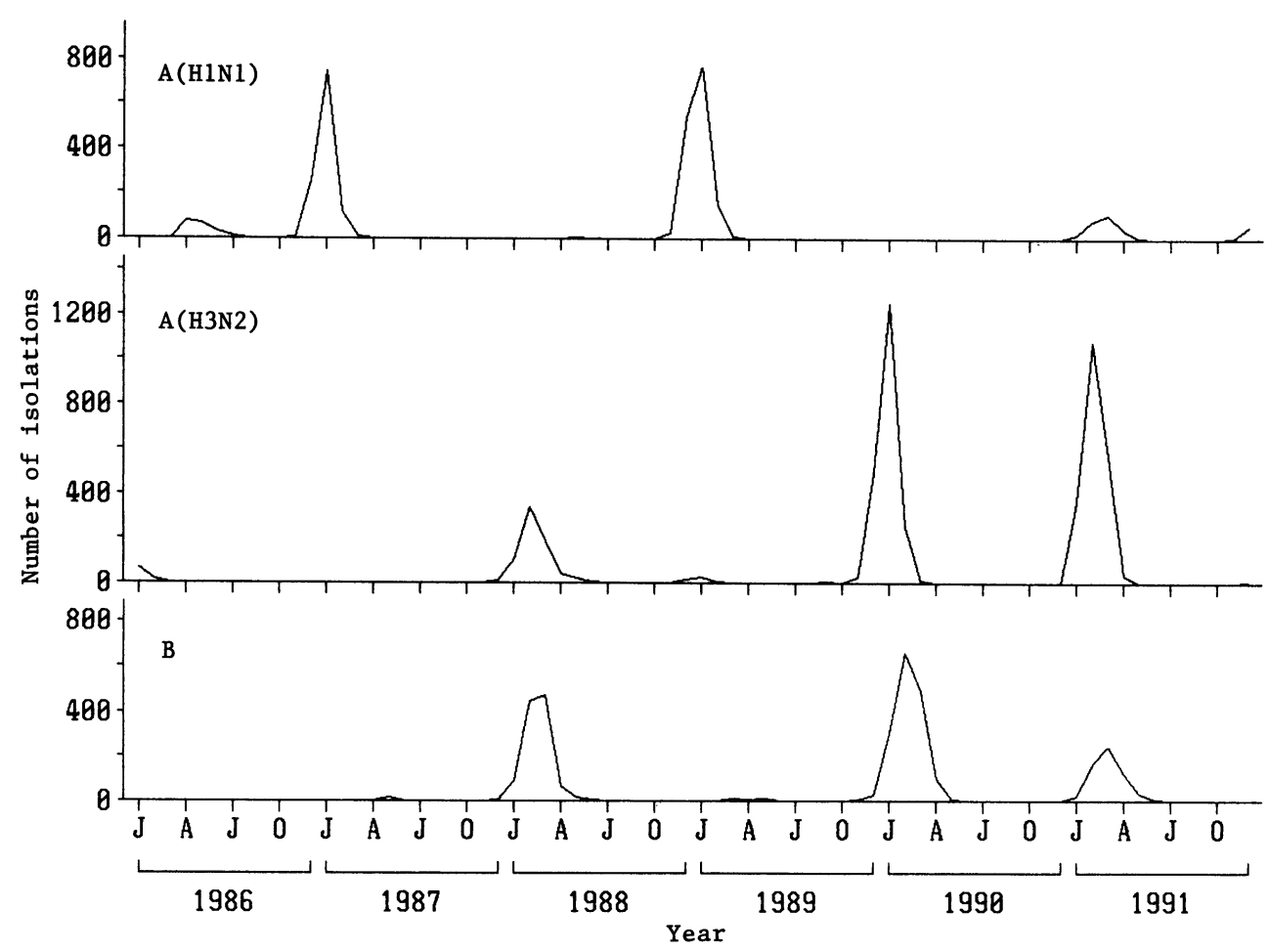

Fig. 24. Reported isolations of influenza viruses from humans, by month, 1986-1991

reported from Sendai National Hospital; other eight institutions reported isolations ranging from one to seven. Isolation patterns were characteristic of each type (Fig. 25). Type 1 was isolated in a small number every year through all seasons. In 1991, 16 isolations were reported. On the other hand, type 2 isolations fluctuated from year to year: 27 were isolated in 1991 comparing with 34 in 1990. Type 3 was reported every year, peaking in summer. In 1991, 30 were isolated peaking in May. No type 4 isolation was reported. For 1991, 56 (77.8\%) of the total parainfluenza reports were from 0-4 years of age, 12 from 5-9 years, three from 10-14 years, and one from 60s years. Forty-seven (64.4\%) were reported to have had upper respiratory illness, and $12(16.4 \%)$ were associated with lower respiratory illness/pneumonia. 
Vol. 45, Suppl.

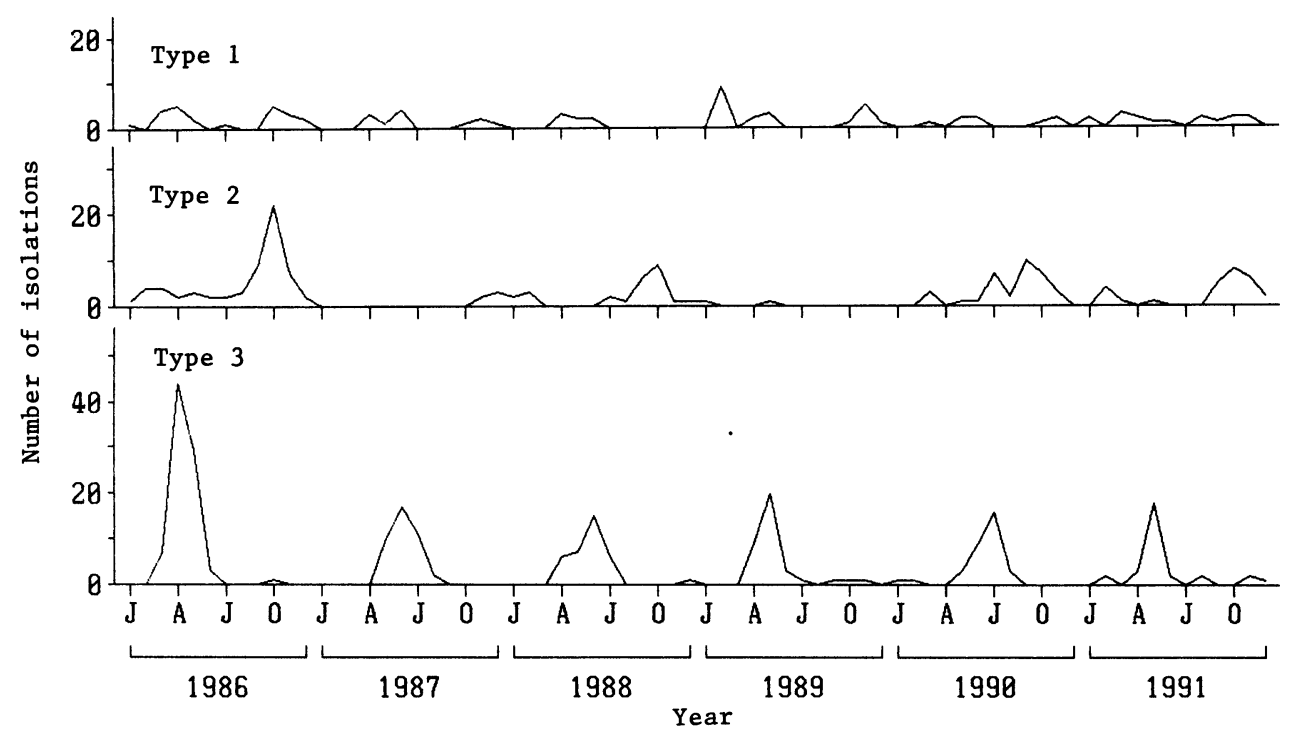

Fig. 25. Reported isolations of parainfluenza viruses from humans, by month, 1986-1991

\section{Mumps virus, RS virus and Measles virus}

A total of 225 mumps virus isolations were reported in 1991 (Fig. 26). Eighteen strains (8.0\%) were isolated from nasopharyngeal specimens of viruspositive cases and 212 strains (94.2\%) from CSF. Among 193 isolations with information on the clinical conditions, $156(80.8 \%)$ were associated with meningitis. No mumps virus isolation from those over 10 years of age was reported. Following the introduction of MMR (Measles-Mumps-Rubella) vaccine in late 1988, occurrence of MMR-associated mumps meningitis became an object of social concern. The Ministry of Health and Welfare issued a notice which indicated that the isolates from CSF of suspected vaccine-associated meningitis cases should be subjected to the strain-differentiation test at NIH. Therefore, the reports included these isolations. Of the mumps virus isolations in 1991, 142 were reported to be of this category.

The number of reports of respiratory syncytial (RS) virus was 127 in 1991. Among these, 69 (54.3\%) were reported from two private clinical laboratories, 42 (33.1\%) from two national hospitals and 16 (12.6\%) from three PHIs. Most RS virus isolations were reported in the winter season. Of the isolates, $122(96.1 \%)$ 


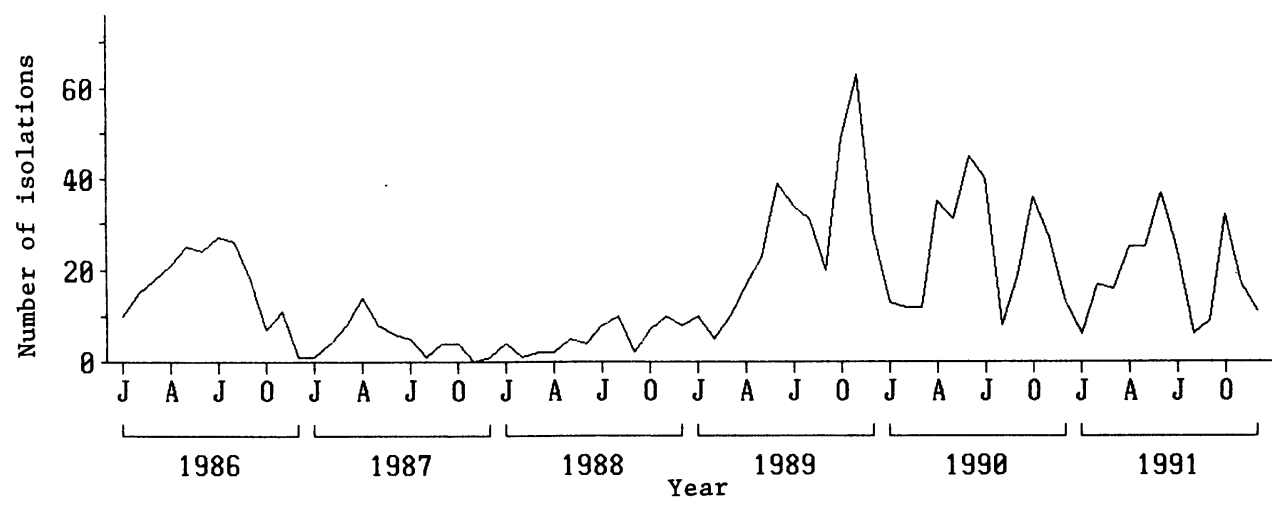

Fig. 26. Reported isolations of mumps virus from humans, by month, 1986-1991

were from children under 5 years of age; 41 were isolated from children, one year of age and 40 were from infants whose ages ranged from less than one month to 11 months. Among 83 reports with clinical information, 33 (39.8\%) were of lower respiratory illness/pneumonia, while 34 cases $(41.0 \%)$ were associated with upper respiratory infections. The majority of $\mathrm{RS}$ isolates were recovered from nasopharyngeal specimens and two were isolated from lung/bronchus materials. One hundred and twenty-two (96.1\%) were isolated in cell culture and six (4.7\%) were identified by ELISA.

Nineteen measles virus isolations were reported in 1991 in April-September peaking in May. Thirteen were from children of 0 to 4-year-old, two from 8-yearold and one each from 6-13- and 15-year-old. Of these, five isolations were associated with lower respiratory illness/pneumonia.

\section{Rubella virus}

Twelve rubella virus isolations were reported in 1991 (Fig. 27). Eleven were reported by Tottori PHI and one by a private clinical laboratory in April-October. They were isolated from the nasopharyngeal specimens of children ranging from 8 months to 13 years old.

\section{Reovirus}

In 1991, one isolation of type 2 reovirus from feces of a 5 -year-old girl was reported by Aichi PHI in June. 
Vol. 45, Suppl.

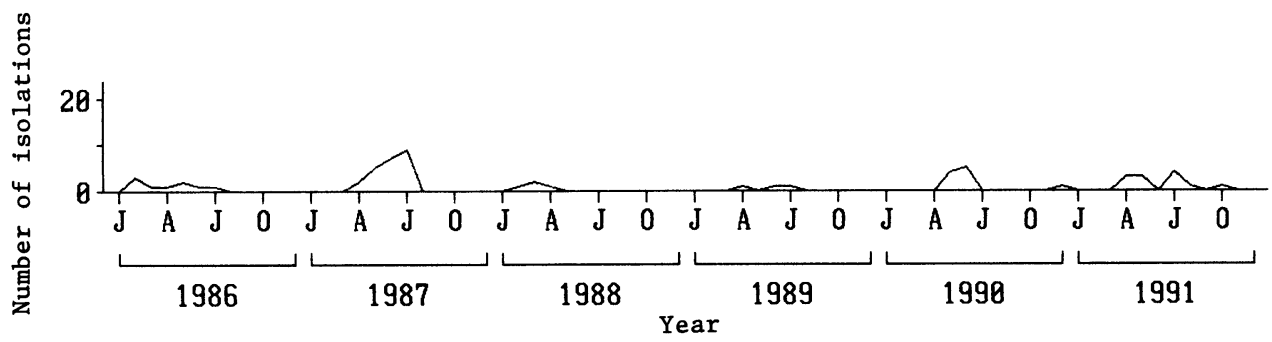

Fig. 27. Reported isolations of rubella virus from humans, by month, 1986-1991

\section{$\underline{\text { Rotavirus }}$}

Annual reports of rotavirus detections peaked in 1986 and have decreased thereafter. In 1991, 582 rotavirus detections were reported with a figure of almost the same level as that in 1990 (524), which was nearly $1 / 2$ of the 1989's 1,054 . The decrease coincided with the case reports from sentinel clinics under the National Epidemiological Surveillance of Infectious Diseases, in which incidence of "infantile vomiting and diarrhea" was markedly low in 1991.

Of the rotavirus reports, $33.2 \%$ were detected by electron microscopy, $46.0 \%$ by ELISA, $22.7 \%$ by R-PHA, in comparison with $34.9 \%, 27.1 \%$ and $20.0 \%$, respectively, in the previous year. In addition, detections by latex agglutination kits were reported in 88 and by polyacrylamide gel electrophoresis (PAGE) in seven cases.

The detection of rotavirus was reported mainly in the winter season and showed the regular yearly pattern, peaking in January-February (Fig. 28). A few detections, however, were reported in the summer season; 31 detections were reported in May-October, 1991.

Rotavirus infections were reported mainly in infants and young children. In 1991, 162 cases (28.4\%) were among those aged less than 12 months and 228 (40.0\%) among 1-year-old. In addition, 123 detections were from those of 2-4 years of age, 44 from 5-14 years, three from 15-19 years, four from 20s years and six from 50 s years. 


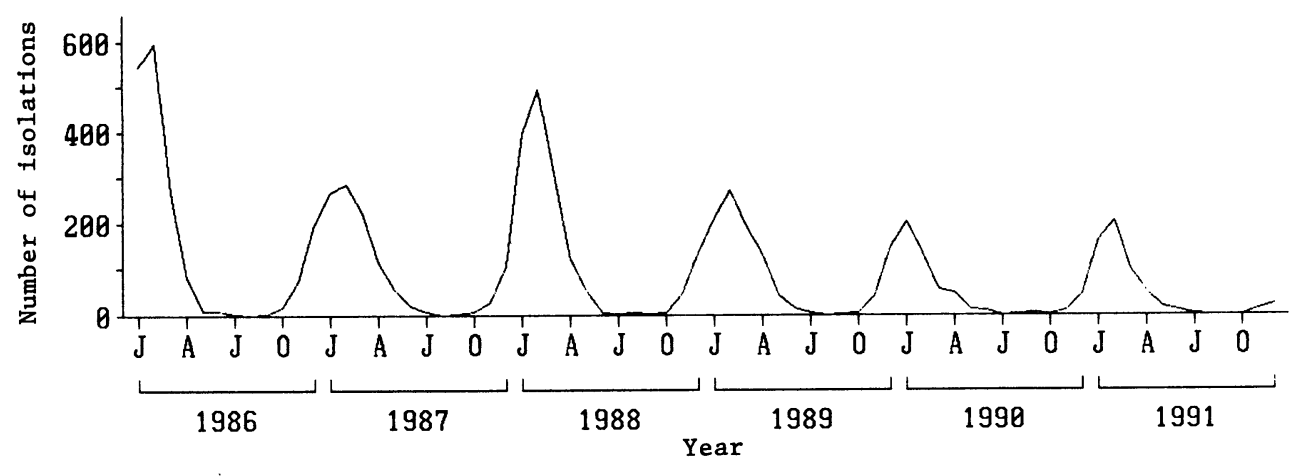

Fig. 28. Reported detections of rotavirus from humans, by month, 19861991

Eight detections in 1991 were of group $C$ rotavirus: These were reported by Tokyo PHI. The virus was detected from an outbreak in a primary school in April by electron microscopy, ELISA and PAGE.

\section{Small round virus (SRV)}

In 1991, 161 cases associated with SRV were reported by 10 laboratories; this represented $103.9 \%$ of the 1990 detections. Of these, 77 reports (47.8\%) were received from Tokyo PHI. Eighty-seven reports were specified as Norwalk-like virus, 14 as calici virus and the other 60 were SRV not specified. All detections were made from feces; 155 (96.3\%) were detected by electron microscopy and six (3.7\%) by ELISA. Eighty SRV detections were reported in January-March, other 32 in April-June, other two in August and the other 47 in October-December. Among 131 SRV reports in 1991 with age information, 94 (71.8\%) were detected from children younger than 15 years of age. The other 37 detections were reported from a wide age distribution, ranging from 15 to 60 s years of age.

\section{Hepatitis A virus}

No detection of hepatitis A virus was reported in 1991. 
Vol. 45, Suppl.

\section{Adenovirus}

In 1991, 1,864 adenovirus isolations including 17 enteric adenovirus, type 41, were reported. The figure represented $116.4 \%$ of that reported in the preceding year. Of these adenovirus isolations, the number of type 3 fluctuated markedly from year to year, resulting in a changing pattern of total adenovirus reports. In 1991, type 3 reports numbered 687, being on the same level as the previous year. Adenovirus type 4, which had decreased in number after the peak in 1984 (307 isolations), turned to increase in 1990 (134), and the second highest number ever since, 229, was recorded in 1991 . Adenovirus type 8 reports had two peaks in annual number, in 1984 (192) and 1988 (131); relatively high number, 91, was reported in 1991. Type 11 isolations decreased to 19 from the previous year's 47 , and type 19 reports numbered, 39 being on the same level as the previous year, while type 37 isolations were 86 , the highest number ever recorded. In general, isolations of adenoviruses peaked in summer, but a fair number was reported throughout the year (Fig. 29).

Four adenovirus types, 1, 2, 5 and 6, have similar characteristics in clinical presentations. The major clinical symptoms reported were fever (67-78\%), inflammation of the upper respiratory tract $(60-66 \%)$ and gastrointestinal tract illness (15-19\%). Reflecting the clinical symptoms, these four types were isolated from the nasopharynx in $74-80 \%$ of virus-positive cases, with the second most frequent isolations from feces $(20-27 \%)$.

For adenovirus type 3 , high association with fever and upper respiratory illness was reported, representing $\mathbf{7 0 . 7 \%}$ and $57.1 \%$ of cases, respectively, and isolations were from the nasopharyn $x$ in $76.0 \%$ of virus-positive cases. In addition, eye diseases were associated with $34.8 \%$ of total isolations of type 3 , and $22.9 \%$ of isolations were from eye specimens.

The tendency of association with eye diseases was more pronounced for type 4 than for type 3. In 1991, the eye disease-association frequency of type 4 was $73.8 \%$ of 206 cases with clinical information, while upper respiratory illness was $23.8 \%$ and gastroenteritis $1.0 \%$. The frequency of type 4 isolation from eye specimens was $68.6 \%$ of the total type 4 isolations, while $31.0 \%$ were recovered from nasopharynx and $1.3 \%$ from feces.

For adenovirus types 8,19 and 37 , most cases with clinical information were suffering from eye diseases; all these viruses except four type 8 and one type 19 were isolated from eye specimens in 1991.

In 1991, isolations of adenovirus type 11 numbered 19. Of these cases, only eight had clinical information: two were associated with eye diseases and five with 
Jpn. J. Med. Sci. Biol., 1992

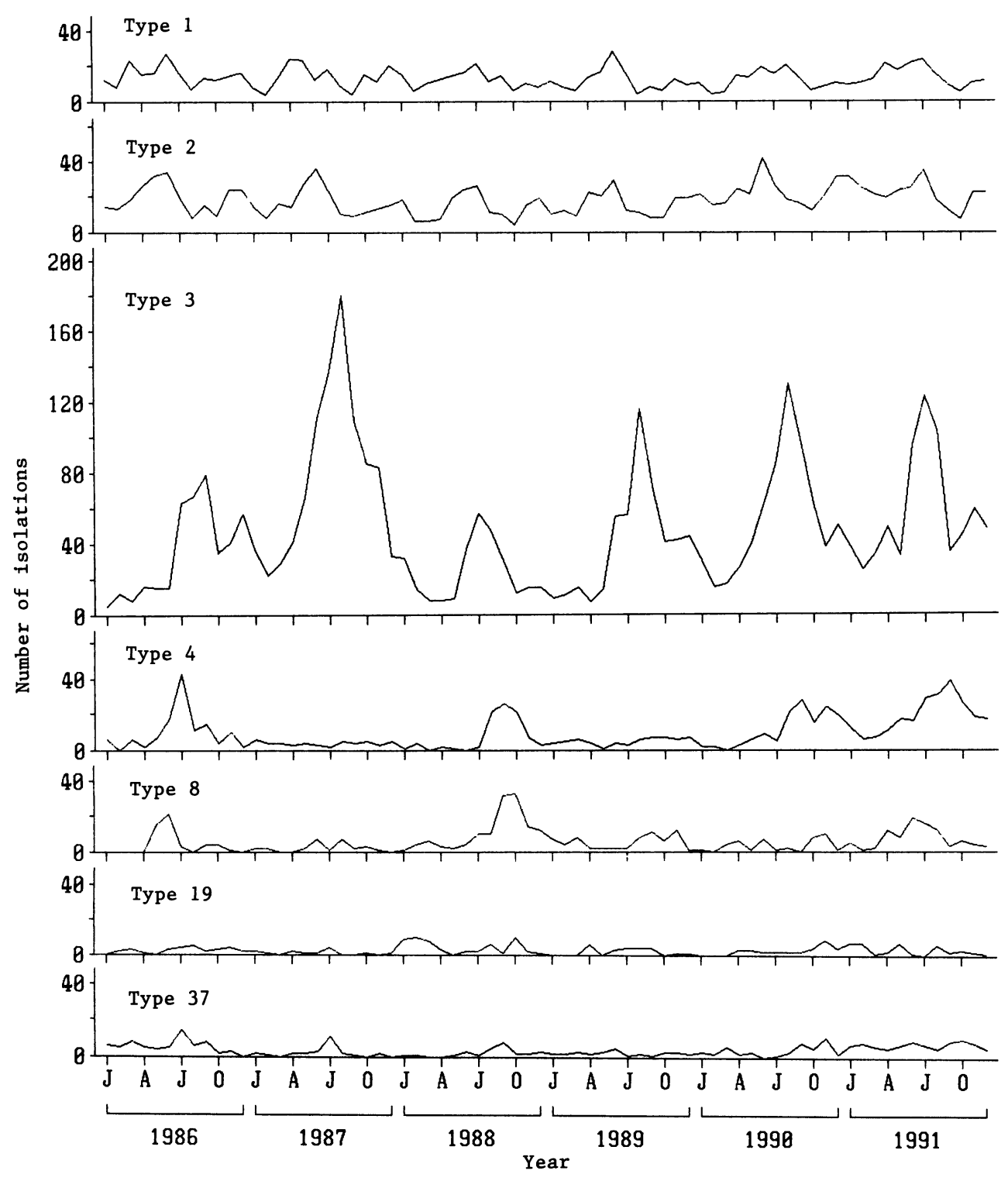

Fig. 29. Reported isolations of adenoviruses from humans, by month, 1986-1991 
Vol. 45, Suppl.

genitourinary disease. Fifteen (78.9\%) of 19 cases were reported to have yielded the virus from urine, three (15.8\%) from eye specimens, one $(5.3 \%)$ from nasopharyngeal specimens and none from feces.

Most patients from whom type 1, 2, 5 or 6 was isolated were young: More than $76 \%$ of the patients with age information were 0-4 years of age. For adenovirus type 3 , isolations from $0-4$ years of age decreased to $39.4 \%$, while $38.1 \%$ were from $5-9$ years of age and only $22.5 \%$ were 10 years old or older. On the other hand, $63.2 \%$ of patients with type 4 isolations were 10 years old or over. Patients infected with adenovirus types 8,19 or 37 were predominantly adults; specifically, reports from those aged 15 years or older were higher than $81 \%$ of respective isolations. Usually, type 11 was isolated from both children and adults, predominating those aged 15 years or older representing $55.6 \%$ of the cases.

Adenoviruses were often associated with inflammation of the lower respiratory tract/pneumonia. In 1991, $111(6.7 \%)$ isolations of the total adenovirus types were reported to be associated with these symptoms.

In 1991, 17 isolations of type 41, one of the enteric adenoviruses, were detected in feces of children ranging from 4 months to 7 years of age. The isolations were scattered in almost every month. The reports were from four PHIs in Tokyo, Aichi, Kagawa and Ehime. Nine were isolated in cell culture and eight by ELISA.

All type-identified adenoviruses were isolated in cell culture except eight enteric adenoviruses which were detected by ELISA. Among the adenoviruses detected in 1991, 112 were type-unknown; 84 were detected by cell culture, 19 by electron microscopy, three by ELISA and 10 by latex agglutination.

\section{Herpes simplex virus}

In 1991, 673 herpes simplex virus (HSV) isolations were reported. Of these, 410 (60.9\%) were from PHIs, 72 (10.7\%) from national hospitals and 191 (28.4\%) from private clinical laboratories. The number of reports with typing results was 567 (84.2\% of the total HSV reports): 466 (82.2\%) were type 1, and the other 101 (17.8\%) were type 2. The figures were almost the same as the previous year's (Fig. 30).

Major isolation materials for type 1 were nasopharyngeal specimens in 315 $(67.9 \%)$, followed by skin/vesicle in $93(20.0 \%)$, genital sources including skin/vesicle of genital herpes in 34 (7.3\%) and eye specimens in 16 (3.4\%). Other isolation specimens were four CSF, three oral mucosa and one each of feces, blood, 
Jpn. J. Med. Sci. Biol., 1992

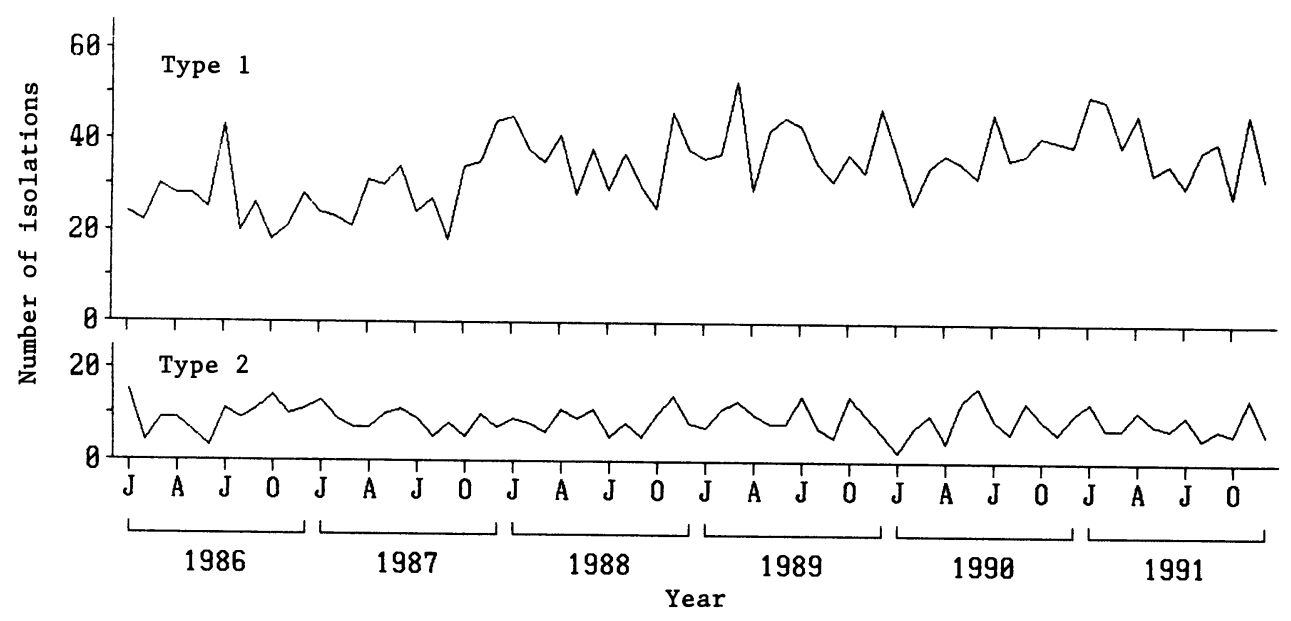

Fig. 30. Reported isolations of herpes simplex viruses from humans, by month, 1986-1991

brain and liver. On the other hand, the majority of type $2 \mathrm{HSV}$ were isolated from genital sources in $65(65.0 \%)$ and from skin/vesicle specimens in 29 (29.0\%); only four were isolated from nasopharyngeal specimens and two from urine.

Descriptive clinical symptoms and signs were reported in 365 cases for type 1. Patients with type 1 isolations were reported to have fever (58.1\%), inflammation of the upper respiratory tract $(44.1 \%)$, stomatitis $(41.6 \%)$, vesicles $(17.0 \%)$, exanthem $(12.6 \%)$ and genitourinary disease $(5.5 \%)$. Other clinical symptoms reported were gastrointestinal illness $(6.8 \%)$, herpangina $(4.7 \%)$ and eye diseases (4.1\%). Among 56 type 2 reports with clinical information, genitourinary infection was reported in $40(71.4 \%)$. Other clinical symptoms reported were vesicle formation in 28, exanthem in nine cases and fever in two cases.

Four cases with type 1 isolation were associated aseptic meningitis and two encephalitis. One each case with meningitis and encephalomyelitis was related with type-unidentified HSV isolation. No type 2 isolation with central nervous system involvement was reported in 1991 (Table).

Isolations from clinically diagnosed genital herpes cases included 23 type 1 and 59 type 2.

The major method for detection of HSV 1 was virus isolation in cell culture (94.4\%). In 1991, however, 28 (6.0\%) type 1 and 34 (33.7\%) type 2 viruses were detected directly from clinical specimens by the fluorescent antibody technique (FA). 
Vol. 45, Suppl.

\section{Varicella-Zoster virus and Cytomegalovirus}

The majority of reports of Varicella-Zoster virus (VZV) and cytomegalovirus (CMV) were from a small number of institutions, particularly private clinical laboratories. Yearly numbers of isolations of these two viruses are on almost the same levels. Forty-two isolations of VZV were reported in 1991. Of these, 23 were reported from private clinical laboratories, 17 were from a national hospital and two from PHIs. All were isolated from skin/vesicle sources.

Isolation of 381 strains of CMV was reported. Of these, 310 (81.4\%) were from private clinical laboratories, 68 were from national hospitals and three were from a PHI. Isolations from patients younger than 12 months of age numbered 154 , and those from 1-year-old children 59, representing $40.8 \%$ and $15.6 \%$, respectively, of the reports for which ages were known. The isolation specimens reported were mainly urine, accounting for 213 (56.2\%), nasopharyngeal specimens, 161 (42.5\%), and lung/bronchi 10 (2.6\%). In addition one each isolation from CSF and pancreas specimen was reported in 1991.

\section{Rickettsia tsutsugamushi}

Two R. tsutsugamushi isolation from humans were reported for 1991. These were isolated in March from blood of scrub typhus patients having residence in suburbs of Tokyo. Mice were used for isolation.

\section{Chlamydia}

Isolation reports of Chlamydia under this reporting system started in 1986. A total of 376 cases were reported from 11 institutions for 1991. Among the isolation reports, 189 (50.3\%) were detected by FA, 94 (25.0\%) were diagnosed by ELISA and 115 (30.6\%) were isolated in cell culture. Since the ELISA kit available at present does not differentiate between Chlamydia trachomatis (C. trachomatis) and Chlamydia psittaci (C.psittaci), the detection of chlamydia by ELISA is shown as "Chlamydia not typed" in the tables in this publication. However, on analysis, it could be considered as $C$. trachomatis when recovered from genitourinary sources.

Among 369 reports with age information (240 males and 129 females), 24 cases (6.5\%) were reported from the group of $15-19$ years of age, 163 cases (44.2\%) from $20-29$ years, 90 (24.4\%) from $30-39$ years and 78 (21.1\%) from $40-49$ years. No isolation from those under 15 years of age was reported in 1991.

Of these isolations, $360(95.7 \%)$ were from genital sources and $12(3.2 \%)$ from urine. Six detections from eye specimens of adults (20-40 years of age) were 
reported by Sapporo City PHI and Kumamoto PHI. No isolation from nasopharyngeal sources was reported.

\section{Mycoplasma}

It is known that atypical pneumonia due to mycoplasma breaks out every four years in Japan. The most recent periodic increase peaked in 1988, when a total of 234 isolations of $M$. pneumoniae were reported from six PHIs. In 1991, the number of reports was only two. These were isolated by Kawasaki City PHI from nasopharyngeal specimens of children ( 5 and 7 years of age) with atypical pneumonia in December.

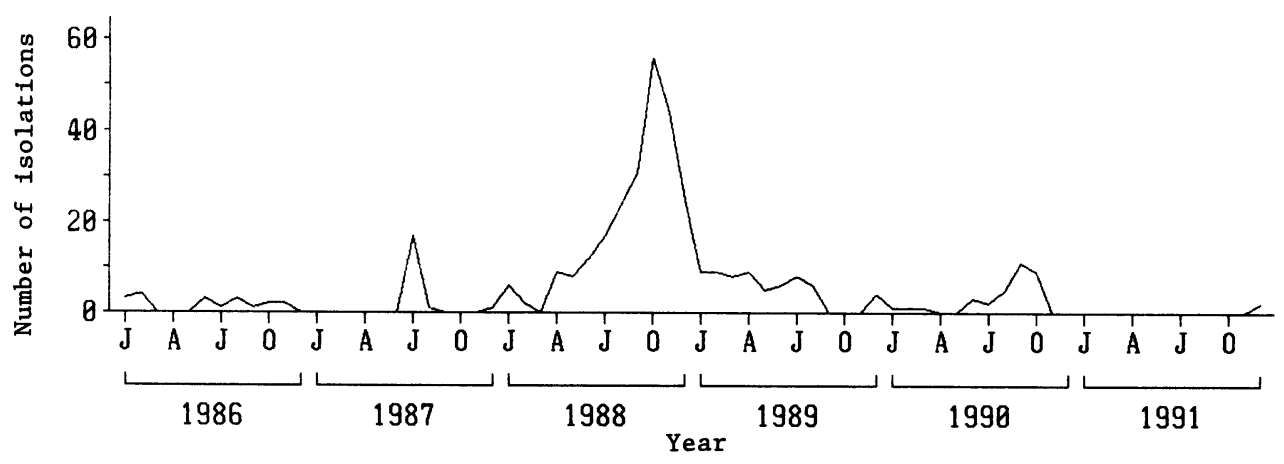

Fig. 31. Reported isolations of $M$. pneumoniae from humans, by month, 1986-1991 
Vol. 45, Suppl.

3-2. Isolation of viruses and rickettsia from non-human sources

Only PHIs report isolation of viruses from non-human sources. A total of 63 isolations of viruses from non-human sources were reported in 1991 by three PHIs. Most of these isolations were carried out through the survey programs conducted by prefectures or cities.

Of these, 32 enterovirus isolations were from environmental sources such as sewage: Coxsackievirus types B1 (three isolations), B2 (10), B3 (three), B5 (four), echovirus types 7 (two), 9 (one), 30 (one) and poliovirus types 2 (four) and 3 (three). While coxsackievirus types B1 and B2 were isolated in the later half of the year, types B3, B5 and echovirus in the early three months.

In addition, one adenovirus type 2 was isolated in October.

Other 30 isolations were of Japanese encephalitis $(\mathrm{JE})$ virus reported by two PHIs; Osaka (29 isolations) and Kyoto City (one). Twenty-seven JE viruses were isolated in August, other two in July and the other one in September, 1991. All JE viruses were isolated from mosquitoes. Among 30 virus-positive specimens, one provided virus-positive result in mice and 29 in cell culture.

No. R. tsutsugamushi from non-human sources was reported in 1991. 